\title{
Nanoliposome-Encapsulated Brinzolamide-hydropropyl- $\beta$-cyclodextrin Inclusion Complex: A Potential Therapeutic Ocular Drug-Delivery System
}

\section{OPEN ACCESS}

Edited by:

Jianxun Ding,

Changchun Institute of Applied

Chemistry (CAS), China

Reviewed by:

Yanqi Ye,

The University of North Carolina at Chapel Hill, United States

Tianjiao Ji,

Boston Children's Hospital, Harvard University, United States

*Correspondence:

Xiangrong Song

songxr@scu.edu.cn

Jinhui Wu

wujinhui@scu.edu.cn

tThese authors have contributed equally to this work.

Specialty section:

This article was submitted to Experimental Pharmacology and Drug

Discovery,

a section of the journal

Frontiers in Pharmacology

Received: 30 December 2017

Accepted: 26 January 2018

Published: 13 February 2018

Citation:

Wang F, Bao X, Fang A, Li H, Zhou Y, Liu Y, Jiang C, Wu J and

Song $X$ (2018)

Nanoliposome-Encapsulated

Brinzolamide-hydropropyl-

$\beta$-cyclodextrin Inclusion Complex:

A Potential Therapeutic Ocular

Drug-Delivery System.

Front. Pharmacol. 9:91.

doi: 10.3389/fphar.2018.00091
Fazhan Wang ${ }^{1 \dagger}$, Xingting Bao ${ }^{1 \dagger}$, Aiping Fang ${ }^{1,2+}$, Huili Li ${ }^{1}$, Yang Zhou ${ }^{1}$, Yongmei Liu ${ }^{1}$ Chunling Jiang ${ }^{1}$, Jinhui $W_{u^{1 *}}$ and Xiangrong Song ${ }^{1 *}$

1 State Key Laboratory of Biotherapy, Geriatrics and Cancer Center, West China Hospital and Collaborative Innovation Center for Biotherapy, Sichuan University, Chengdu, China, ${ }^{2}$ West China School of Public Health, Sichuan University, Chengdu, China

Novel ocular drug delivery systems (NODDSs) remain to be explored to overcome the anatomical and physiological barriers of the eyes. This study was to encapsulate brinzolamide (BRZ)-hydropropyl- $\beta$-cyclodextrin (HP- $\beta$-CD) inclusion complex (HP$\beta-C D / B R Z)$ into nanoliposomes and investigate its potential as one of NODDS to improve BRZ local glaucomatous therapeutic effect. HP- $\beta-C D / B R Z$ was firstly prepared to enhance the solubility of poorly water-soluble BRZ. The HP- $\beta-C D / B R Z$ loaded nanoliposomes (BCL) were subsequently constructed by thin-film dispersion method. After the optimization of the ratio of BRZ to HP- $\beta-C D$, the optimal BCL showed an average size of $82.29 \pm 6.20 \mathrm{~nm}$, $\zeta$ potential of $-3.57 \pm 0.46 \mathrm{mV}$ and entrapment efficiency (EE) of $92.50 \pm 2.10 \%$ with nearly spherical in shape. The X-ray diffraction (XRD) confirmed the formation of HP- $\beta-C D / B R Z$ and $B C L$. The in vitro release study of $B C L$ was evaluated using the dialysis technique, and $B C L$ showed moderate sustained release. BCL (1 mg/mL BRZ) showed a 9.36-fold increase in the apparent permeability coefficient and had a sustained and enhanced intraocular pressure reduction efficacy when compared with the commercially available formulation (BRZ-Sus) (10 mg/mL BRZ). In conclusion, BCL might have a promising future as a NODDS for glaucoma treatment.

Keywords: brinzolamide, cyclodextrin, inclusion complex, nanoliposomes, intraocular pressure, ocular drug delivery

\section{INTRODUCTION}

Despite easy accessibility for topical drug administration, overcoming the anatomical and physiological barriers of the eye remains one of the greatest challenges for ocular drug delivery (Bucolo et al., 2012; Zhang et al., 2015). Drug retention is impeded by tear reflex, blinking, and nasolacrimal drainage (Morrison et al., 2013). Cornea barrier protects the eye from the passage of any foreign molecules including drugs into the eye, and thus only a small fraction of the topically applied drug penetrates the cornea and reaches intraocular tissues (Mun et al., 2014). As a result, repeated dosing is needed, which increases the patient discomfort and other adverse effects (Luo et al., 2011). A system which behaves like a solution and at the same time can lead to retention of drug in the eye and increase corneal permeability is of great urgency. 
Nanoliposomes, as biodegradable, biocompatible and nonimmunogenic drug delivery system, have been widely applied (van Rooijen and van Nieuwmegen, 1980; Mugabe et al., 2006; Arias, 2013; Sercombe et al., 2015; Ji et al., 2017). They can encapsulate hydrophilic drugs into the inner aqueous phase, whereas entrap hydrophobic drugs into the hydrophobic lipid phase (Allen and Cullis, 2004; Chen et al., 2009). However, it is difficult to obtain high drug-loading capacity in the lipid phase because of the limited space offered by the lipid phase, and a large amount of hydrophobic drugs can destabilize the structural integrity of the liposomal layers (Song et al., 2012). Inclusion complexes are one of the most useful strategies in the pharmaceutical field, which can solubilize a wide range of hydrophobic drugs (Morrison et al., 2013; Zhang et al., 2013; Alomrani et al., 2014; Ji et al., 2016; Maria et al., 2017; Soliman et al., 2017). The binding force between the guest drug molecule and the cyclodextrin host molecule is usually weak, possibly leading to rapid dissociation of the inclusion complexes due to dilution by plasma or extracellular fluids (Chen et al., 2014). Encapsulation of hydrophobic drugs in the form of inclusion complexes into nanoliposomes has been investigated as a new strategy to combine the advantages of inclusion complexes and nanoliposomes, namely the drug-incyclodextrin-in-nanoliposome (DCL) system (Piel et al., 2006; Hatzi et al., 2007; Zhang et al., 2015). The presence of cyclodextrin in the aqueous compartment of nanoliposomes would not affect the characteristics of conventional liposomes but prolong drug release compared to conventional liposomes (Maestrelli et al., 2010; Zhang et al., 2015). Although a number of studies on DCL systems have been reported (Dhule et al., 2012; Rahman et al., 2012; Lapenda et al., 2013; Alomrani et al., 2014), to date, the therapeutic application of DCL systems as an ocular delivery system remains to be explored.

Nanoliposome systems are applicable in ocular delivery, which have the potential of increasing corneal permeability and improving retention time (de Sa et al., 2015; Li et al., 2016). Moreover, some functional liposomes have been constructed (Eriksen et al., 2017; Huang et al., 2017; Tan et al., 2017). Cyclodextrin, with a hydrophilic outer surface and a cavity at its center and low renal toxicity, has the ability to form hydrophilic inclusion complexes by a molecular complexation with a lot of hydrophobic drugs (Ji et al., 2016; Maria et al., 2016, 2017; Abd El-Gawad et al., 2017). Hydropropyl- $\beta$-cyclodextrin (HP- $\beta$-CD), a type of cyclodextrin, is commonly used for ocular drug delivery and has been approved by the FDA (Bozkir et al., 2012). Many in vitro and in vivo studies also showed that the inclusion complex of poor water-soluble drugs by HP- $\beta$-CD could increase drug solubility, corneal permeability, and ophthalmic bioavailability (Ito et al., 2013; Morrison et al., 2013). Encouraged by these previous results, we postulated that DCL might have the potential to enhance ocular permeation of the hydrophobic model drug brinzolamide (BRZ) and enhance its therapeutic efficacy for glaucoma.

Previously, we have used inclusion complex and nanoliposomes to encapsulate the low water-soluble drug BRZ, respectively. Both formulations improved the corneal permeation and therapeutic efficacy of BRZ to some extent
(Zhang et al., 2013; Li et al., 2016). Here, we further combined $\mathrm{HP}-\beta-\mathrm{CD}$ and nanoliposomes to prepare BRZ-HP- $\beta-\mathrm{CD}$ inclusion complex (HP- $\beta$-CD/BRZ)-loaded nanoliposomes (BCL) as shown in Figure 1. The pharmaceutical properties including UV/vis spectra, particle size, surface charge, entrapment efficiency (EE) of BRZ, X-ray diffraction (XRD), and in vitro release profile were then characterized systemically. The ex vivo corneal permeability, in vivo intraocular pressure (IOP) reduction efficiency and preliminary safety of BCL were finally evaluated.

\section{MATERIALS AND METHODS}

\section{Materials}

BRZ (purity > 99\%) was obtained from Dalian Meilun Biology Technology Company (Dalian, China). HP- $\beta-C D$ (purity $>99 \%$, DS = 7) was supplied by Xi'an Deli Chemicals Corporation (Xi'an, China). Soybean phosphatidylcholine (SPC) was provided by Shanghai A.V.T. Pharmaceutical, Co., Ltd. (Shanghai, China). Cholesterol was purchased from Shanghai Yuanju Biology Technology Company (Shanghai, China). The commercial formulation AZOPT ${ }^{\circledR}$ (BRZ-Sus) was supplied by Alcon Laboratories (Puurs, Belgium). All the other reagents were of analytical grade unless otherwise stated.

\section{Animals}

White New Zealand rabbits (male, 2.0-2.5 kg) were used for each formulation $(n=6)$. Animals were housed individually and allowed free access to food and water with a 12:12 h cyclic lighting schedule. After a week of adaptation, animals were admitted to experiments. All animal experiments were approved and supervised by the State Key Laboratory of Biotherapy Animal Care and Use Committee (Sichuan University, Chengdu, Sichuan, China).

\section{Preparation of $\mathbf{H}-\beta-\mathrm{CD} / \mathrm{BRZ}$ Inclusion Complex}

According to previously described methods by Zhang et al. (2013), the inclusion complex was prepared with some

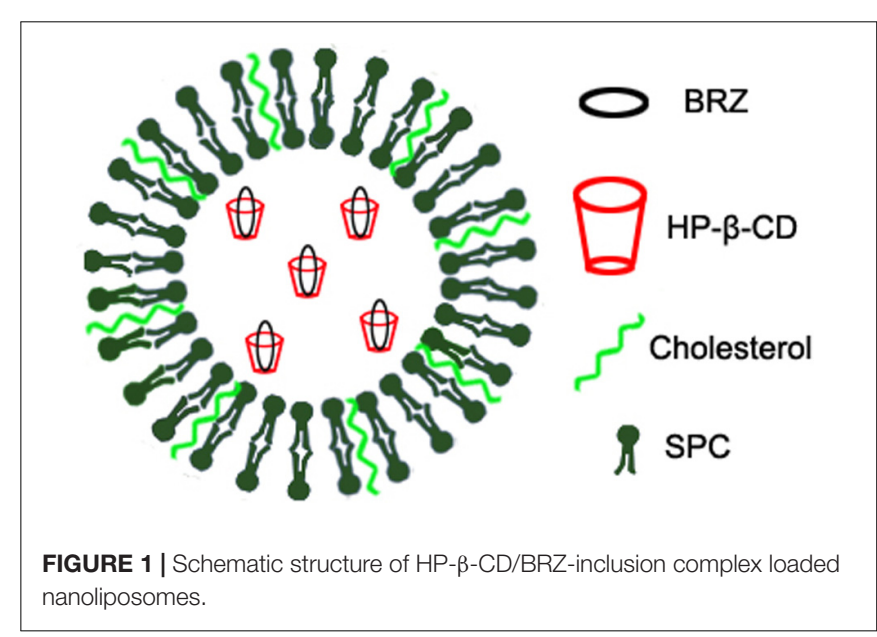


modifications. Briefly, BRZ and HP- $\beta$-CD at different molar ratio $(2: 1,1: 1,1: 2$, and 1:3) were dissolved by ethanol in a roundbottomed flask. After $1 \mathrm{~h}$ of ultrasound at $37^{\circ} \mathrm{C}$, the ethanol was removed using a rotary evaporator in vacuum. To dissolve the inclusion complex, PBS was added to the flask followed by filtering through a $0.22 \mu \mathrm{m}$ membrane. The content of BRZ in the inclusion complex was analyzed by high-performance liquid chromatography (HPLC, Waters, Milford, MA, United States) at $252 \mathrm{~nm}$. The mobile phase, at $1 \mathrm{~mL} / \mathrm{min}$ flow rate, was composed of acetonitrile and water at a volume ratio of 40:60.

\section{Preparation of BCL}

BCL was prepared using a modified thin-film dispersion method (Lapenda et al., 2013; Li et al., 2016). Briefly, SPC and cholesterol were dissolved in a mixture solvent of chloroform/methanol $(4: 1, \mathrm{v} / \mathrm{v})$. The organic solvents were evaporated using a rotary evaporator at $37^{\circ} \mathrm{C}$, and then the formed thin film was further dried under high vacuum for $1 \mathrm{~h}$. The lipid film was hydrated with HP- $\beta$-CD/BRZ inclusion complexes at $60^{\circ} \mathrm{C}$ for $1 \mathrm{~h}$ to obtain a suspension. To obtain BCL, the above suspension was sonicated for $3 \mathrm{~min}$ at $100 \mathrm{~W}$ in an ice bath and filtered through a $0.22-\mu \mathrm{m}$ membrane filter.

The BRZ-loaded nanoliposomes (LP/BRZ) were obtained using the similar procedure with the addition of BRZ into the chloroform/methanol $(4: 1 \mathrm{v} / \mathrm{v})$ solvent mixture and hydrating lipid film with sterile PBS. The blank liposomes (Blank LP) were also prepared using the similar preparation process of LP/BRZ without adding BRZ.

\section{Characterization of BCL Ultraviolet-Visible (UV/vis) Spectroscopy}

The UV/vis absorption spectra of BRZ, HP- $\beta-C D$, Blank LP, inclusion complex, LP/BRZ, and BCL were recorded using a SHIMADZU UV-2600 UV/vis spectrophotometer in the range of 200-600 nm.

\section{Particle Size and $\zeta$ Potential}

The average particle size, size distribution [polydispersity index (PDI)], and $\zeta$ potential of diluted BCL were recorded by Zetasizer Nano ZS90 (Malvern Instruments, Malvern, United Kingdom). All measurements were carried out at $25^{\circ} \mathrm{C}$ after $3 \mathrm{~min}$ of equilibration and were conducted in triplicate. All the data were presented as mean \pm standard deviation (SD).

\section{Morphology}

The morphological examination of BCL was performed by transmission electron microscopy (TEM, H-600, Hitachi, Japan). Briefly, a drop of liposomal suspension was placed onto copper electron microscopy grids, and then they were negatively stained with $2 \%$ phosphotungstic acid for observation at an acceleration voltage of $100 \mathrm{kV}$.

\section{Drug Entrapment Efficiency}

According to previously described methods (Rahman et al., 2012), the drug content of BCL was determined by separating BCL from the unentrapped drug using cooling centrifugation at $50,000 \mathrm{rpm}$ for $30 \mathrm{~min}$ at a temperature of $4^{\circ} \mathrm{C}$. Following the removal of the supernatant, $1 \mathrm{ml}$ of acetonitrile was added into the BCL sediment and sonicated for 20 min to extract BRZ from BCL. After centrifugation at 13,000 rpm for $10 \mathrm{~min}$, the content of BRZ in the supernatant diluted with acetonitrile was quantified by HPLC as described previously. Analysis was performed in triplicate and the values were expressed as mean \pm SD. EE was calculated by the following calculation equation:

$$
\mathrm{EE}=\text { (amount of BRZ in BCL/initial BRZ amount) } \times 100 \% .
$$

\section{Stability of BCL}

The optimized formulation was stored at $4^{\circ} \mathrm{C}$ for 2 weeks in order to investigate the preliminary stability. In the test, the physicochemical characteristics such as particle size and EE were recorded and changes over time were evaluated.

\section{XRD Analysis}

$\mathrm{X}$-ray diffraction was performed to investigate the crystal structure according to the former study (Tao et al., 2017). Different samples (pure BRZ, HP- $\beta-C D, H P-\beta-C D / B R Z$, and $\mathrm{BCL}$ ) were examined using an X-ray diffractometer (X'Pert Pro Philips, Netherlands) at a voltage of $40 \mathrm{kV}$ and a current of $40 \mathrm{~mA}$. The scans were carried out at a scanning rate of $10^{\circ} \mathrm{C} / \mathrm{min}$ in the $2 \theta$ range from 0 to $50^{\circ} \mathrm{C}$.

\section{In Vitro BRZ Release from BCL}

In vitro release studies were carried out using the dynamic dialysis technique in sink conditions. Briefly, $2 \mathrm{~mL}$ of different formulations containing the drug were placed into different dialysis bags (molecular weight cut-off $=3,500 \mathrm{Da}$ ) and immersed in $40 \mathrm{~mL}$ of simulated tear fluid (STF) and continuously agitated in an orbital shaker maintained at $37^{\circ} \mathrm{C}$. At given time intervals, $1 \mathrm{~mL}$ of receptor phase was removed and replaced with an equal volume of STF. The drug concentration was quantified by the HPLC method as described above. All data were presented as mean \pm SD of three independent measurements.

\section{Corneal Permeability}

The transcorneal permeability of BCL was evaluated using a Franz diffusion chamber which consisted of a donor and a receiver compartment (with a volume of 1.0 and $2.0 \mathrm{~mL}$, respectively). The diffusion chamber was maintained at a constant temperature $\left(37 \pm 0.2^{\circ} \mathrm{C}\right)$ by a thermostatic water bath, under mixing conditions using a magnetic stirrer. The cornea together with a $2-\mathrm{mm}$ ring of sclera was excised immediately after the rabbits $(n=3)$ were sacrificed and stored in glutathione bicarbonate ringer (GBR) buffer after rinsing with cold saline before use. One $\mathrm{mL}$ of the GBR solution was added to the endothelial (receptor) side of the cornea, while $0.5 \mathrm{~mL}$ of different formulations was added to the epithelial (donor) side of the cornea. Different samples from the receptor chamber were withdrawn at $0,0.5,1,1.5,2,3,4,5$ and $6 \mathrm{~h}$ and replaced with fresh GBR buffer-receptor solution. The content of drug permeation across the cornea was determined by the HPLC method as described above. The experiment was done in triplicate.

The apparent permeation coefficient $\left(\mathrm{P}_{\mathrm{app}}, \mathrm{cm} / \mathrm{s}\right)$ of each formulation was calculated using the following equation 
(Goskonda et al., 2000):

$$
\mathrm{P}_{\mathrm{app}}=\left(1 / \mathrm{AC}_{0}\right)^{*}(\mathrm{dM} / \mathrm{dt})
$$

where A is the available corneal surface area for diffusion $\left(\mathrm{cm}^{2}\right)$, $\mathrm{C}_{0}$ is the initial drug concentration $(\mu \mathrm{g} / \mathrm{mL})$ in the donor compartment, and dM/dt is the flux across the cornea $\left(\mu \mathrm{g} / \mathrm{cm}^{2} \mathrm{~h}\right)$.

\section{Corneal Hydration Level}

According to the literature (Vega et al., 2008), the corneal hydration level (HL) was evaluated by measuring total water content by the gravimetric method. At the end of the corneal permeability study $(n=3)$, the wet weight of each cornea $\left(\mathrm{W}_{1}\right)$ and dry corneal weight $\left(\mathrm{W}_{2}\right)$ after a desiccation at $100^{\circ} \mathrm{C}$ for $6 \mathrm{~h}$ were obtained, respectively. The corneal $\mathrm{HL}$ was calculated using the following equation: $\mathrm{HL} \%=\left(\mathrm{W}_{1}-\mathrm{W}_{2}\right) / \mathrm{W}_{1} \times 100 \%$.

\section{In Vivo IOP Measurement}

IOP was recorded in $\mathrm{mmHg}$ with a Tono-pen $\mathrm{XL}^{\circledR}$ tonometer (Reichert, NY, United States) calibrated according to the manufacturer's instructions. IOP was recorded until the animals $(n=6)$ were accustomed to the experimental procedure. Each formulation $(50 \mu \mathrm{L})$ was instilled topically into the right eye, while the left eye received no treatment to minimize the diurnal and individual variations. The IOP was measured at $0.5,1,2,3,4,5,6,8,10,12$ and $24 \mathrm{~h}$ after instilled.

\section{Statistical Analysis}

The data were expressed as mean \pm SD. Statistical significance was assessed by a two-way analysis of variance using SPSS 16.0 (SPSS, Inc., Chicago, IL, United States). The $p$-values $<0.05$ were considered statistically significant difference.

\section{RESULTS}

\section{UV/vis Spectroscopy}

The influence of the BCL on the absorption of BRZ was evaluated by UV/vis spectroscopy. The absorption spectra of BRZ, HP- $\beta-C D$, Blank LP, HP- $\beta-C D / B R Z$ inclusion complex, $\mathrm{LP} / \mathrm{BRZ}$ and BCL in acetonitrile were determined (Figure 2). The spectrum of BCL exhibited a characteristics absorption peak at $252 \mathrm{~nm}$, which was similar to the peak of BRZ. These results suggested that the HPLC method developed for BRZ measurement with a detection wavelength of $252 \mathrm{~nm}$ could also be applied to determine the BRZ concentration in BCL.

\section{Preparation of BCL}

Different ratios of BRZ and $\mathrm{HP}-\beta-\mathrm{CD}$ were used to establish the optimal preparation conditions in which BCL are formed. The drug content of BCL ranged between 84.38 and $92.50 \%$ and the size of BCL ranged between $82.29 \pm 6.20 \mathrm{~nm}$ and $90.62 \pm 11.30 \mathrm{~nm}$ depending on the BRZ/ HP- $\beta$-CD molar ratio (Figure 3A).

\section{Characterization of BCL}

\section{Particle Size, $\zeta$ Potential, and EE}

The physicochemical properties of optimal BCL were evaluated. The particle size of the optimal BCL was $82.29 \pm 6.20 \mathrm{~nm}$ with a narrow size distribution (PDI $=0.21 \pm 0.01$ ) (Figure 3C). The $\zeta$ potential of BCL was $-3.57 \pm 0.46 \mathrm{mV}$ (Figure 3D). The appearance and morphological studies of BCL were also conducted. LP/BRZ and BCL colloidal solution were observed as a slightly blue opalescence with obvious Tyndall effect compared with water (Figures 3E,F). BCL were seen to be distinct lipid membrane structure with a particle size diameter of about $70 \mathrm{~nm}$ (Figure 3G).

\section{Storage Stability}

The particle size and EE of BCL were determined at a predetermined time of storage at $4^{\circ} \mathrm{C}$. BCL displayed good stability with no detectable changes in particle size and $\mathrm{EE}$ for at least 3 days (Figure 3B).

\section{XRD Analysis}

$X$-ray diffractograms were obtained from different samples of BRZ, HP- $\beta-C D, H P-\beta-C D / B R Z$ and BCL to evaluate the crystal structure and entrapment of BRZ in BCL. The X-ray spectrum of BRZ showed several sharp and narrow peaks between 5 and $50^{\circ} \mathrm{C}(2 \theta)$ with a maximal peak at $2 \theta=24.97^{\circ} \mathrm{C}$, indicating their crystalline nature. HP- $\beta$-CD displayed a specific broad peak at $2 \theta=20^{\circ} \mathrm{C}$ in its X-ray diffractogram. Neither HP- $\beta$-CD/BRZ nor $\mathrm{BCL}$ showed the peak at $2 \theta=24.97^{\circ} \mathrm{C}$ (Figure 4).

\section{In Vitro Release Profile}

The in vitro release experiments were performed to investigate the successful inclusion and the sustained release characteristic of BCL. A sustained release phase was observed in BCL within a period of $9 \mathrm{~h}(1-10 \mathrm{~h})$ as presented in Figure 5. BRZ release from the inclusion complex was slightly slower than free drug but faster than LP/BRZ. Moreover, BCL displayed a slower release compared to LP/BRZ.

\section{Corneal Permeation and Corneal Hydration}

The corneal penetration study was performed to evaluate the effect of BCL on transcorneal transportation. The corneal permeation characteristics of BRZ-Sus $(10 \mathrm{mg} / \mathrm{mL}), \mathrm{LP} / \mathrm{BRZ}$ $(1 \mathrm{mg} / \mathrm{mL})$, and BCL $(1 \mathrm{mg} / \mathrm{mL})$ were shown in Figure 6. There was no significant difference in the cumulative permeation amount at $0-2 \mathrm{~h}$ among the three groups. At 3-6 h, BRZ-Sus and BCL showed a significant increase of BRZ cumulative permeation compared with LP/BRZ. A linear relationship was observed for all preparations over the time period from 0.5 to $6 \mathrm{~h}$, indicating that the corneal integrity was maintained throughout the experiment. Moreover, the permeation coefficient of BCL was much larger than those of BRZ-Sus and LP/BRZ (Table 1, $p<0.001$ ). BCL showed a 9.36- and 0.40-fold increase in $\mathrm{P}_{\text {app }}$ when compared with Brz-Sus and LP/BRZ, respectively.

Corneal HL was used to evaluate the safety of BCL (Vega et al., 2008). Its normal value is often from 75 to $80 \%$ (Vega et al., 2008). The corneal HL of BRZ-Sus, LP/BRZ, and BCL were 


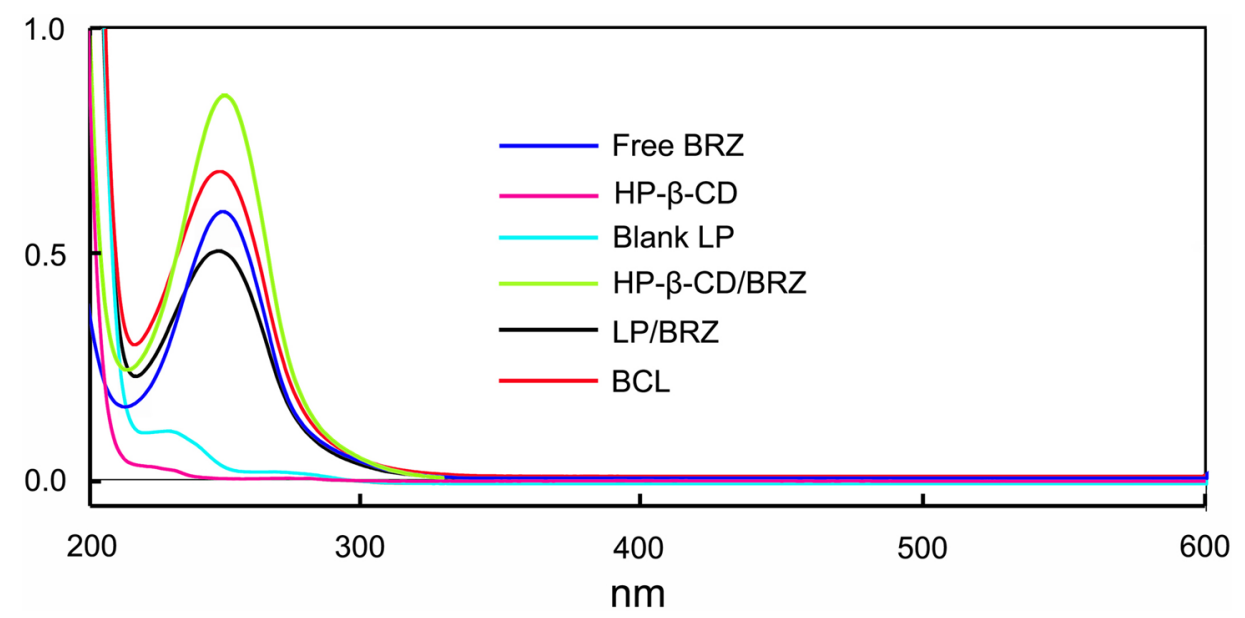

FIGURE 2 | The UV/vis spectra of free BRZ, HP- $\beta-C D$, Blank LP, HP- $\beta-C D / B R Z$ inclusion complex, LP/BRZ, and BCL in acetonitrile.

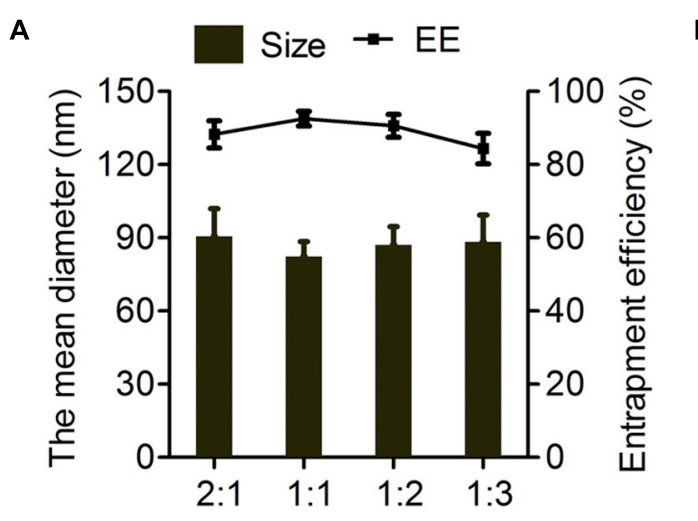

The BRZ/HP- $\beta-C D$ molar ratio

C

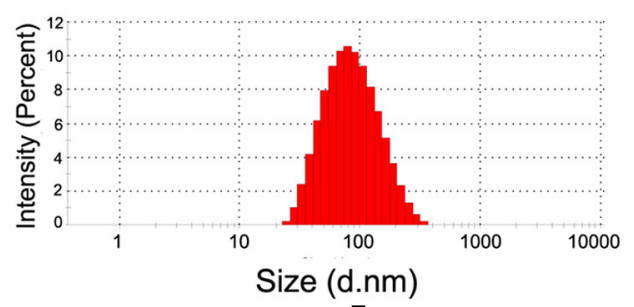

E

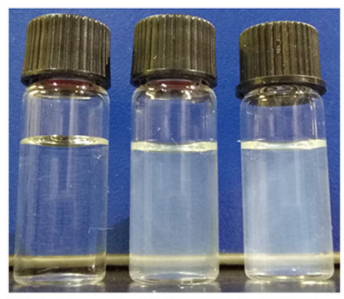

Water LP/BRZ BCL
$F$

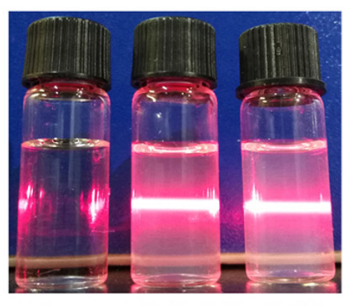

Water LP/BRZ BCL
B Size $\rightarrow$ EE

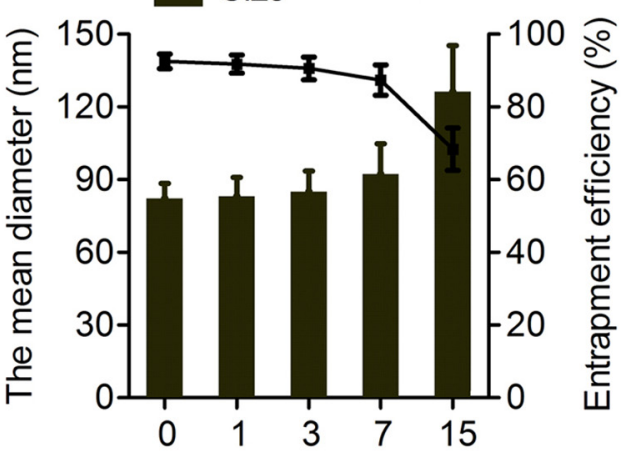

Time (Days)

D Zeta Potential Distribution

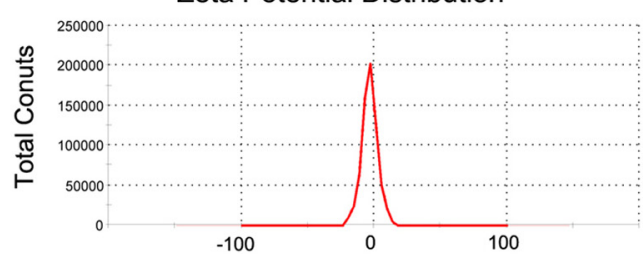

Apparent Zeta Potential $(\mathrm{mV})$

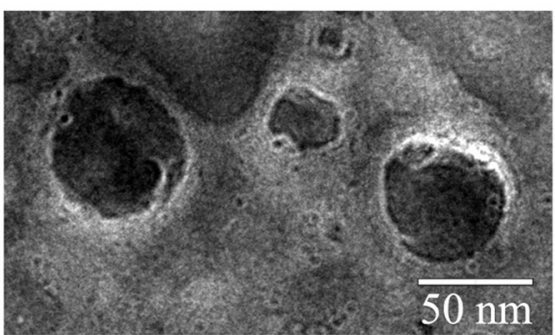

FIGURE 3 | Effects of various HP- $\beta-C D / B R Z$ ratios on diameter and drug entrapment efficiency of BCL (A) $(n=3)$. Change in size and entrapment efficiency of BCL at various time points stored at $4^{\circ} \mathrm{C}(\mathbf{B})(n=3)$. Size distribution $(\mathbf{C})$, $\zeta$ potential $(\mathbf{D})$, appearance $(\mathbf{E})$, and tyndall effect $(\mathbf{F})$ of $B C L$. TEM images of BCL (G). 
$80.12 \pm 2.43 \%, 79.37 \pm 2.19 \%$, and $78.56 \pm 3.04 \%$, respectively. No statistical difference in $\mathrm{HL} \%$ was found $(p>0.05)$, indicating BCL might be safe for topical ocular use.

\section{In Vivo IOP-Lowering Effect}

As shown in Figure 7, BCL (1 mg/mL BRZ) presented more effective IOP reduction with a longer term role than BRZ-Sus (10 $\mathrm{mg} / \mathrm{mL} \mathrm{Brz}$ ) and LP/BRZ (1 $\mathrm{mg} / \mathrm{mL} \mathrm{Brz}$ ). The novel formulation of BRZ had an onset of less than $1 \mathrm{~h}$, achieved a maximum IOP reduction of $32.3 \%$ at $2 \mathrm{~h}$, and sustained an effective IOP reduction until the 12th hour, while Brz-Sus began to result in an effective IOP reduction at $0.5 \mathrm{~h}$ and quickly reached its peak effect at $1 \mathrm{~h}$ (an average reduction of $16.12 \%$ from baseline). Furthermore, IOP was significantly lower in the BCL group at any time point from 2 to $12 \mathrm{~h}$ than Brz-Sus group. Even though the dosage of $\mathrm{BRZ}$ was just $10 \%$ in $\mathrm{BCL}$ compared with BRZ-Sus, the IOP reduction efficacy was much higher.

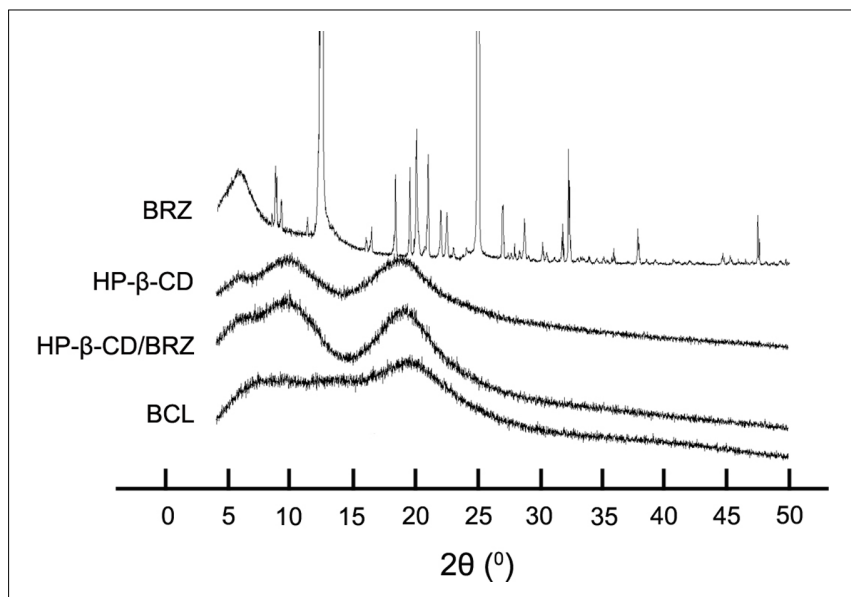

FIGURE 4 |X-ray diffractograms of BRZ, HP- $\beta-C D, H P-\beta-C D / B R Z$, and BCL.

\section{DISCUSSION}

In this work, we aimed to construct a novel ocular delivery system DCL loaded with BRZ (BCL) and investigate its potential to improve BRZ local glaucomatous therapeutic effect. BCL was prepared by hydrating lipid film with inclusion complex (Lapenda et al., 2013), and HP- $\beta$-CD was selected to enhance the solubility of poorly soluble drug BRZ (Zhang et al., 2013). According to the literature, the content of HP- $\beta$-CD was critical for the physicochemical properties of DCL (Chen et al., 2014; Cutrignelli et al., 2014). Cyclodextrins would interact with the lipid membrane and influence the formation of DCL (Cutrignelli et al., 2014). Thus, the amount of HP- $\beta$-CD was optimized based on the particle size and EE of BCL in this study. As seen in Figure 3A, EE first increased and then reached a plateau with the decrease of the BRZ/HP- $\beta$-CD molar ratio while the average diameter of BCL first decreased and then increased. Further increase in the HP- $\beta$-CD content (when the BRZ/HP- $\beta$-CD molar ratio was set at 1:3) cannot correspondingly enhance the BRZ entrapment into BCL but reduced EE. The cholesterol in the lipid bilayer of nanoliposomes might enter the excessive HP- $\beta$-CD cavity (Morrison et al., 2013; Chen et al., 2014), thereby forming unstable nanoliposomes which cannot incorporate enough HP$\beta$-CD/BRZ-inclusion complex. The highest EE and the smallest particle size of BCL were achieved when BRZ and HP- $\beta-C D$ was fed at a 1:1 molar ratio, and thus BCL containing 1:1 $\mathrm{BRZ} / \mathrm{H}-\beta-\mathrm{CD}$ were chosen for the subsequent investigations.

The optimal BCL were nano-sized (less than $100 \mathrm{~nm}$ ) with negative surface charge probably resulting from the phosphatidylcholine head group in the outer nanoliposomes surface (Ascenso et al., 2013). Interestingly, BCL colloidal suspension had a similar appearance, average size, and surface charge to LP/BRZ despite the introduction of HP- $\beta-C D$ (Maestrelli et al., 2010). This phenomenon indicated that HP$\beta$-CD might mainly distribute in the inner aqueous core of the nanoliposomes, which was consistent with the literature (Cavalcanti et al., 2011; Lapenda et al., 2013; Chen et al., 2014). Moreover, it was clear that the peaks of BRZ disappeared from
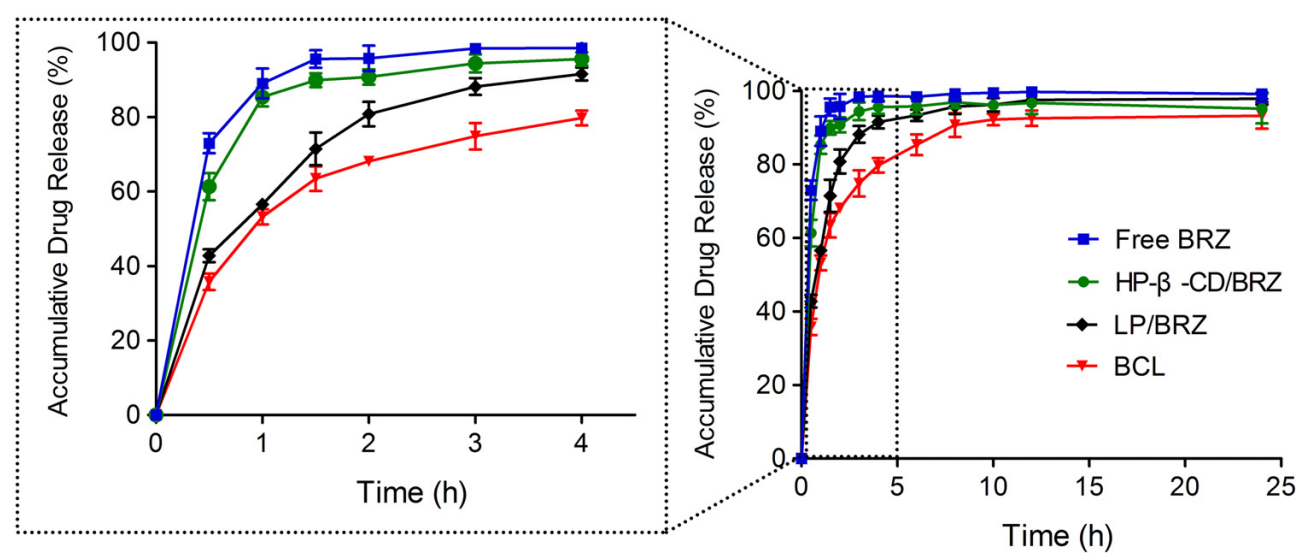

FIGURE 5| In vitro release profiles of BRZ from BRZ solution (free drug), HP- $\beta$-CD/BRZ, LP/BRZ, and BCL at $37^{\circ} \mathrm{C}$ in simulated tear fluid. Data are expressed as mean $\pm \operatorname{SD}(n=3)$. 


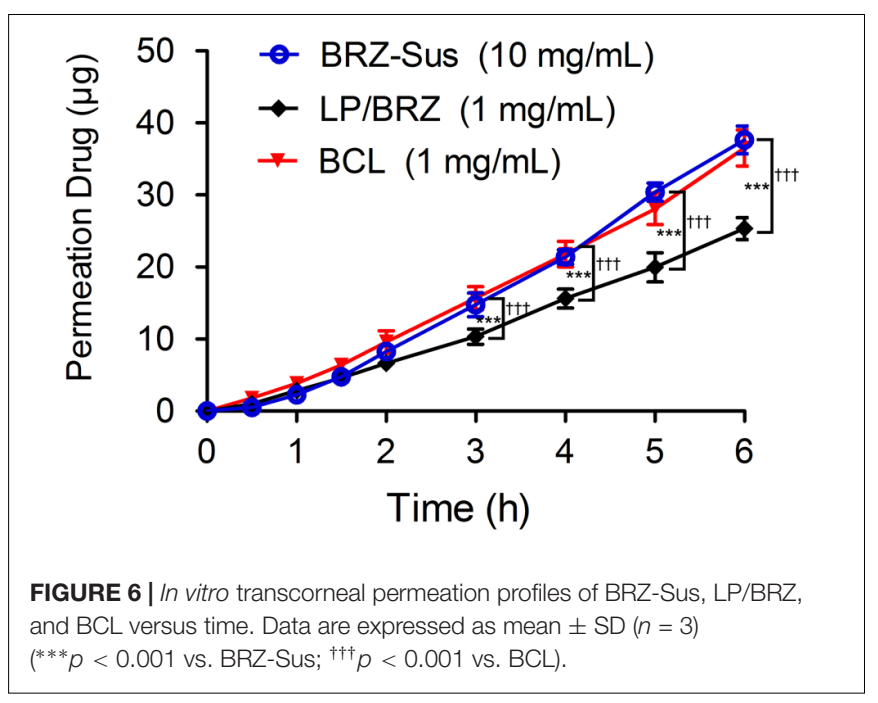

TABLE 1 | Corneal hydration level and $\mathrm{P}_{\text {app }}$ of different formulations containing brinzolamide. Data are expressed as mean $\pm \mathrm{SD}(n=3)$.

\begin{tabular}{lcc}
\hline Formulation & HL $\%$ & $\mathbf{P}_{\text {app }}\left(\times \mathbf{1 0}^{\mathbf{6}} \mathbf{~ c m} \mathbf{s}\right)$ \\
\hline BRZ-Sus & $80.12 \pm 2.43$ & $0.36 \pm 0.02$ \\
LP/BRZ & $79.37 \pm 2.19$ & $2.59 \pm 0.07$ \\
BCL & $78.56 \pm 3.04$ & $3.73 \pm 0.13$ \\
\hline
\end{tabular}

the diffraction pattern of BCL, demonstrating that BRZ was completely and successfully encapsulated into BCL.

BCL could be stable for about 7 days at $4^{\circ} \mathrm{C}$, which might benefit from the negatively charged surface. However, EE sharply declined when BCL were kept for 15 days. The cholesterol in BCL might go into the hydrophobic cavity of $\mathrm{HP}-\beta-\mathrm{CD}$ to compete with BRZ (Lapenda et al., 2013; Morrison et al., 2013), hence partially contributing to partial leakage of BRZ from the inclusion complex and the instability of nanoliposomes. HP- $\beta$-CD has previously been reported to extract cholesterol from cell membranes (Morrison et al., 2013) and decrease the integrity of liposomes composed of cholesterol and saturated phospholipids (Hatzi et al., 2007). The phenomenon found in this study was in line with these former studies. The free BRZ would easily pass through the lipid bilayer of the unstable BCL, which led to the reduced EE after relatively long-term storage. Generally, this kind of destructive role of $\mathrm{HP}-\beta-\mathrm{CD}$ on DCL basically depends on the HP- $\beta$-CD affinity balance between the guest drug molecules and lipid components of nanoliposomes (Piel et al., 2006; Chen et al., 2014). According to the preliminary stability results, BCL would be further developed to be a freezedrying formulation for long-term storage.

BCL performed a slower release than $\mathrm{HP}-\beta-\mathrm{CD} / \mathrm{BRZ}$ inclusion complex and LP/BRZ. It seems that the slower release profile of BCL was partially attributed to the fact that there were more barriers to the diffusion of BRZ from the BCL than LP/BRZ. As for BCL, two definitely different routes may account for the BRZ release (Chen et al., 2014). The whole HP- $\beta-C D / B R Z$ inclusion complex might release from the destructive BCL or transport to lipid phase and then release. For the other way, BRZ might release from the dissociated $\mathrm{HP}-\beta-\mathrm{CD} / \mathrm{BRZ}$ inclusion complex inside BCL followed by diffusion out of the nanoliposomes and dispersion through the dialysis bag (Chen et al., 2014). To date, there still remains a lot of work to do to clarify the relative contributions of the two definitely different routes in release of BCL.

BCL exhibited enhanced and sustained IOP reduction effect as shown in Figure 7. This promising efficacy of BCL on glaucoma treatment may be mainly explained by the combinatorial advantages of BCL with HP- $\beta-C D$ and nanoliposomes. Neutral liposome with a size of about $100 \mathrm{~nm}$ were reported to be safe for ocular applications without ocular irritability (Taniguchi et al., 1988). Moreover, particle size $<10 \mu \mathrm{m}$ was recommended and considered optimal for ophthalmic preparation to minimize eye irritation (Kassem et al., 2007; Yuan et al., 2009). Accordingly, BCL colloidal solution with small size rather than BRZ-Sus might attenuate ocular irritation, thereby reducing the loss of BRZ by tear flushing. In addition, BCL with higher corneal binding affinity (Natarajan et al., 2012) can increase the retention on the surface of cornea, probably extending the absorption of BRZ. BCL displayed significant improvement in corneal permeation in contrast to the conventionally prepared LP/BRZ. This was thought to be the cause of the more efficient IOP lowering effect of BCL. Moreover, the intact HP- $\beta-\mathrm{CD} / \mathrm{BRZ}$ inclusion complex

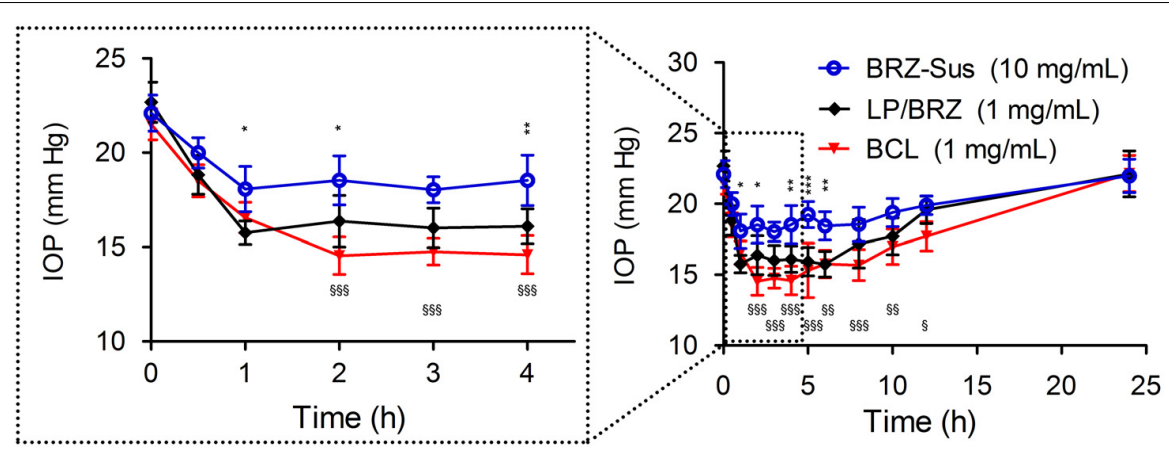

FIGURE 7 | In vivo intraocular pressure (IOP) profiles of white New Zealand rabbits treated with BRZ-Sus, LP/BRZ, and BCL. Data are expressed as mean \pm SD $(n=6)\left({ }^{*} p<0.05,{ }^{* *} p<0.01\right.$, and ${ }^{* * *} p<0.001 \mathrm{vs}$. LP/BRZ; ${ }^{\S} p<0.05,{ }^{\S} p<0.01$, and ${ }^{\S \S} p<0.001 \mathrm{vs}$. BCL). 
released from BCL might lead to the enhanced penetration according to our previous study (Zhang et al., 2013). Of note, the sustained release profile of BCL as shown in the in vitro release study might be linked to the prolonged therapeutic efficacy of BCL.

\section{CONCLUSION}

In this study, a novel drug-in-cyclodextrin-in-nanoliposome system containing BRZ was successfully prepared and its potential application in glaucomatous treatment was investigated. The optimized ratio of BRZ and HP- $\beta-C D$ was set at $1: 1$ to prepare BCL with small size and high drug content. BCL incorporated BRZ very well and showed a sustained release profile and enhanced corneal permeation. Moreover, this new formulation of BRZ achieved an improved and prolonged effect of IOP reduction with similar safety to the commercially available formulation on white New Zealand rabbits. However, further studies are still required to investigate the in vitro release properties of BCL and elucidate how the release profile affects the therapeutic efficacy of BCL. In sum, all the data indicated that

\section{REFERENCES}

Abd El-Gawad, A. E. H., Soliman, O. A., El-Dahan, M. S., and Al-Zuhairy, S. A. S. (2017). Improvement of the ocular bioavailability of econazole nitrate upon complexation with cyclodextrins. AAPS PharmSciTech 18, 1795-1809. doi: 10.1208/s12249-016-0609-9

Allen, T. M., and Cullis, P. R. (2004). Drug delivery systems: entering the mainstream. Science 303, 1818-1822. doi: 10.1126/science.1095833

Alomrani, A. H., Shazly, G. A., Amara, A. A., and Badran, M. M. (2014). Itraconazole-hydroxypropyl-beta-cyclodextrin loaded deformable liposomes: in vitro skin penetration studies and antifungal efficacy using Candida albicans as model. Colloids Surf. B Biointerfaces 121, 74-81. doi: 10.1016/j.colsurfb.2014. 05.030

Arias, J. L. (2013). Liposomes in drug delivery: a patent review (2007 - present). Expert Opin. Ther. Pat. 23, 1399-1414. doi: 10.1517/13543776.2013.828035

Ascenso, A., Cruz, M., Euleterio, C., Carvalho, F. A., Santos, N. C., Marques, H. C., et al. (2013). Novel tretinoin formulations: a drug-in-cyclodextrin-in-liposome approach. J. Liposome Res. 23, 211-219. doi: 10.3109/08982104.2013.78 8026

Bozkir, A., Denli, Z. F., and Basaran, B. (2012). Effect of hydroxypropyl-betacyclodextrin on the solubility, stability and in-vitro release of ciprofloxacin for ocular drug delivery. Acta Pol. Pharm. 69, 719-724.

Bucolo, C., Drago, F., and Salomone, S. (2012). Ocular drug delivery: a clue from nanotechnology. Front. Pharmacol. 3:188. doi: 10.3389/fphar.2012.00188

Cavalcanti, I. M., Mendonca, E. A., Lira, M. C., Honrato, S. B., Camara, C. A., Amorim, R. V., et al. (2011). The encapsulation of beta-lapachone in 2-hydroxypropyl-beta-cyclodextrin inclusion complex into liposomes: a physicochemical evaluation and molecular modeling approach. Euro. J. Pharm. Sci. 44, 332-340. doi: 10.1016/j.ejps.2011.08.011

Chen, J., Lu, W. L., Gu, W., Lu, S. S., Chen, Z. P., Cai, B. C., et al. (2014). Drug-incyclodextrin-in-liposomes: a promising delivery system for hydrophobic drugs. Expert Opin. Drug Deliv. 11, 565-577. doi: 10.1517/17425247.2014.884557

Chen, Y., Lu, Y., Chen, J., Lai, J., Sun, J., Hu, F., et al. (2009). Enhanced bioavailability of the poorly water-soluble drug fenofibrate by using liposomes containing a bile salt. Int. J. Pharm. 376, 153-160. doi: 10.1016/j.ijpharm.2009. 04.022

Cutrignelli, A., Lopedota, A., Denora, N., Laquintana, V., Tongiani, S., and Franco, M. (2014). Characterization and release studies of liposomal gels containing glutathione/cyclodextrins complexes potentially useful for cutaneous administration. J. Pharm. Sci. 103, 1246-1254. doi: 10.1002/jps.23900
DCL might be one of the potential ocular delivery systems for hydrophobic drugs and BCL were worthy of further investigation as a novel anti-glaucoma formulation candidate of BRZ.

\section{AUTHOR CONTRIBUTIONS}

XS conceived of the study. JW designed the experiments. XS and FW wrote the manuscript. FW and XB conducted most of the experiments. AF performed the statistical analysis and drafted the manuscript. HL and $\mathrm{YZ}$ performed all the preliminary experiments. YL and CJ participated in literature research and manuscript editing. All authors reviewed and approved the manuscript.

\section{ACKNOWLEDGMENTS}

This work was financially supported by the Sichuan Province Science and Technology Support Program (Grant Nos. 2015SZ0234 and 2017GZ0413) and the National Natural Science Foundation of China (Grant No. 81600006).

de Sa, F. A., Taveira, S. F., Gelfuso, G. M., Lima, E. M., and Gratieri, T. (2015). Liposomal voriconazole (VOR) formulation for improved ocular delivery. Colloids Surf. B Biointerfaces. 133, 331-338. doi: 10.1016/j.colsurfb.2015.06.036

Dhule, S. S., Penfornis, P., Frazier, T., Walker, R., Feldman, J., Tan, G., et al. (2012). Curcumin-loaded gamma-cyclodextrin liposomal nanoparticles as delivery vehicles for osteosarcoma. Nanomedicine 8, 440-451. doi: 10.1016/j.nano.2011. 07.011

Eriksen, A. Z., Brewer, J., Andresen, T. L., and Urquhart, A. J. (2017). The diffusion dynamics of PEGylated liposomes in the intact vitreous of the ex vivo porcine eye: a fluorescence correlation spectroscopy and biodistribution study. Int. J. Pharm. 522, 90-97. doi: 10.1016/j.ijpharm.2017.03.003

Goskonda, V. R., Hill, R. A., Khan, M. A., and Reddy, I. K. (2000). Permeability of chemical delivery systems across rabbit corneal (SIRC) cell line and isolated corneas: a comparative study. Pharm. Dev. Technol. 5, 409-416. doi: 10.1081/ PDT- 100100557

Hatzi, P., Mourtas, S., Klepetsanis, P. G., and Antimisiaris, S. G. (2007). Integrity of liposomes in presence of cyclodextrins: effect of liposome type and lipid composition. Int. J. Pharm. 333, 167-176. doi: 10.1016/j.ijpharm.2006.09.059

Huang, Y., Tao, Q., Hou, D., Hu, S., Tian, S., Chen, Y., et al. (2017). A novel ion-exchange carrier based upon liposome-encapsulated montmorillonite for ophthalmic delivery of betaxolol hydrochloride. Int. J. Nanomed. 12, 1731-1745. doi: 10.2147/IJN.S122747

Ito, Y., Nagai, N., Okamoto, N., Shimomura, Y., Nakanishi, K., and Tanaka, R. (2013). Reduction of enhanced rabbit intraocular pressure by instillation of pyroglutamic acid eye drops. Biol. Pharm. Bull. 36, 1017-1023. doi: 10.1248/ bpb.b13-00159

Ji, T., Lang, J., Wang, J., Cai, R., Zhang, Y., Qi, F., et al. (2017). Designing liposomes to suppress extracellular matrix expression to enhance drug penetration and pancreatic tumor therapy. ACS Nano 11, 8668-8678. doi: 10.1021/acsnano. $7 \mathrm{~b} 01026$

Ji, T., Li, S., Zhang, Y., Lang, J., Ding, Y., Zhao, X., et al. (2016). An MMP-2 responsive liposome integrating antifibrosis and chemotherapeutic drugs for enhanced drug perfusion and efficacy in pancreatic cancer. ACS Appl. Mater. Interfaces 8, 3438-3445. doi: 10.1021/acsami.5b11619

Kassem, M. A., Abdel Rahman, A. A., Ghorab, M. M., Ahmed, M. B., and Khalil, R. M. (2007). Nanosuspension as an ophthalmic delivery system for certain glucocorticoid drugs. Int. J. Pharm. 340, 126-133. doi: 10.1016/j.ijpharm.2007. 03.011

Lapenda, T. L., Morais, W. A., Almeida, F. J., Ferraz, M. S., Lira, M. C., Santos, N. P., et al. (2013). Encapsulation of trans-dehydrocrotonin in liposomes: an 
enhancement of the antitumor activity. J. Biomed. Nanotechnol. 9, 499-510. doi: 10.1166/jbn.2013.1554

Li, H., Liu, Y., Zhang, Y., Fang, D., Xu, B., Zhang, L., et al. (2016). Liposomes as a novel ocular delivery system for brinzolamide: in vitro and in vivo studies. AAPS PharmSciTech 17, 710-717. doi: 10.1208/s12249-015-0382-1

Luo, Q., Zhao, J., Zhang, X., and Pan, W. (2011). Nanostructured lipid carrier (NLC) coated with Chitosan Oligosaccharides and its potential use in ocular drug delivery system. Int. J. Pharm. 403, 185-191. doi: 10.1016/j.ijpharm.2010. 10.013

Maestrelli, F., Gonzalez-Rodriguez, M. L., Rabasco, A. M., Ghelardini, C., and Mura, P. (2010). New "drug-in cyclodextrin-in deformable liposomes" formulations to improve the therapeutic efficacy of local anaesthetics. Int. J. Pharm. 395, 222-231. doi: 10.1016/j.ijpharm.2010.05.046

Maria, D. N., Abd-Elgawad, A. H., Soliman, O. A., El-Dahan, M. S., and Jablonski, M. M. (2017). Nimodipine ophthalmic formulations for management of glaucoma. Pharm. Res. 34, 809-824. doi: 10.1007/s11095-017-2110-X

Maria, D. N., Mishra, S. R., Wang, L., Abd-Elgawad, A. H., Soliman, O. A., El-Dahan, M. S., et al. (2016). Water-soluble complex of curcumin with cyclodextrins: enhanced physical properties for ocular drug delivery. Curr. Drug Deliv. 14, 875-886.

Morrison, P. W., Connon, C. J., and Khutoryanskiy, V. V. (2013). Cyclodextrinmediated enhancement of riboflavin solubility and corneal permeability. Mol. Pharm. 10, 756-762. doi: 10.1021/mp3005963

Mugabe, C., Azghani, A. O., and Omri, A. (2006). Preparation and characterization of dehydration-rehydration vesicles loaded with aminoglycoside and macrolide antibiotics. Int. J. Pharm. 307, 244-250. doi: 10.1016/j.ijpharm.2005.10.005

Mun, E. A., Morrison, P. W., Williams, A. C., and Khutoryanskiy, V. V. (2014). On the barrier properties of the cornea: a microscopy study of the penetration of fluorescently labeled nanoparticles, polymers, and sodium fluorescein. Mol. Pharm. 11, 3556-3564. doi: 10.1021/mp500332m

Natarajan, J. V., Ang, M., Darwitan, A., Chattopadhyay, S., Wong, T. T., and Venkatraman, S. S. (2012). Nanomedicine for glaucoma: liposomes provide sustained release of latanoprost in the eye. Int. J. Nanomedicine 7, 123-131. doi: $10.2147 /$ IJN.S25468

Piel, G., Piette, M., Barillaro, V., Castagne, D., Evrard, B., and Delattre, L. (2006). Betamethasone-in-cyclodextrin-in-liposome: the effect of cyclodextrins on encapsulation efficiency and release kinetics. Int. J. Pharm. 312, 75-82. doi: 10.1016/j.ijpharm.2005.12.044

Rahman, S., Cao, S., Steadman, K. J., Wei, M., and Parekh, H. S. (2012). Native and beta-cyclodextrin-enclosed curcumin: entrapment within liposomes and their in vitro cytotoxicity in lung and colon cancer. Drug Deliv. 19, 346-353. doi: 10.3109/10717544.2012.721143

Sercombe, L., Veerati, T., Moheimani, F., Wu, S. Y., Sood, A. K., and Hua, S. (2015). Advances and challenges of liposome assisted drug delivery. Front. Pharm. 6:286. doi: 10.3389/fphar.2015.00286

Soliman, O. A. E., Mohamed, E. A. M., El-Dahan, M. S., and Khatera, N. A. A. (2017). Potential use of cyclodextrin complexes for enhanced stability, anti-inflammatory efficacy, and ocular bioavailability of loteprednol etabonate. AAPS PharmSciTech 18, 1228-1241. doi: 10.1208/s12249-0160589-9

Song, C. K., Balakrishnan, P., Shim, C. K., Chung, S. J., Chong, S., and Kim, D. D. (2012). A novel vesicular carrier, transethosome, for enhanced skin delivery of voriconazole: characterization and in vitro/in vivo evaluation. Colloids Surf. B Biointerfaces 92, 299-304. doi: 10.1016/j.colsurfb.2011.12.004

Tan, G., Yu, S., Pan, H., Li, J., Liu, D., Yuan, K., et al. (2017). Bioadhesive chitosanloaded liposomes: a more efficient and higher permeable ocular delivery platform for timolol maleate. Int. J. Biol. Macromol. 94, 355-363. doi: 10.1016/ j.ijbiomac.2016.10.035

Taniguchi, K., Yamamoto, Y., Itakura, K., Miichi, H., and Hayashi, S. (1988). Assessment of ocular irritability of liposome preparations. J. Pharmacobiodyn. 11, 607-611. doi: 10.1248/bpb1978.11.607

Tao, W., Zhu, X., Yu, X., Zeng, X., Xiao, Q., Zhang, X., et al. (2017). Black phosphorus nanosheets as a robust delivery platform for cancer theranostics. Adv. Mater. 29:1603276. doi: 10.1002/adma.201603276

van Rooijen, N., and van Nieuwmegen, R. (1980). Liposomes in immunology: multilamellar phosphatidylcholine liposomes as a simple, biodegradable and harmless adjuvant without any immunogenic activity of its own. Immunol. Commun. 9, 243-256. doi: 10.3109/08820138009065997

Vega, E., Gamisans, F., Garcia, M. L., Chauvet, A., Lacoulonche, F., and Egea, M. A. (2008). PLGA nanospheres for the ocular delivery of flurbiprofen: drug release and interactions. J. Pharm. Sci. 97, 5306-5317. doi: 10.1002/jps. 21383

Yuan, J., Zhai, J. J., Chen, J. Q., Ye, C. T., and Zhou, S. Y. (2009). Preparation of $0.05 \%$ FK506 suspension eyedrops and its pharmacokinetics after topical ocular administration. J. Ocul. Pharmacol. Ther. 25, 345-350. doi: 10.1089/jop.2008. 0125

Zhang, L., Zhang, Q., Wang, X., Zhang, W., Lin, C., Chen, F., et al. (2015). Drug-incyclodextrin-in-liposomes: a novel drug delivery system for flurbiprofen. Int. J. Pharm. 492, 40-45. doi: 10.1016/j.ijpharm.2015.07.011

Zhang, Y., Ren, K., He, Z., Li, H., Chen, T., Lei, Y., et al. (2013). Development of inclusion complex of brinzolamide with hydroxypropyl-beta-cyclodextrin. Carbohydr. Polym. 98, 638-643. doi: 10.1016/j.carbpol.2013.06.052

Conflict of Interest Statement: The authors declare that the research was conducted in the absence of any commercial or financial relationships that could be construed as a potential conflict of interest.

Copyright (c) 2018 Wang, Bao, Fang, Li, Zhou, Liu, Jiang, Wu and Song. This is an open-access article distributed under the terms of the Creative Commons Attribution License (CC BY). The use, distribution or reproduction in other forums is permitted, provided the original author(s) and the copyright owner are credited and that the original publication in this journal is cited, in accordance with accepted academic practice. No use, distribution or reproduction is permitted which does not comply with these terms. 